\title{
Envelope instability in DNA adenine methylase mutants of Salmonella enterica
}

\author{
M. Graciela Pucciarelli, ${ }^{1}$ Ana I. Prieto, ${ }^{2}$ Josep Casadesús ${ }^{2}$ \\ and Francisco García-del Portillo'
}

Author for correspondence: Francisco García-del Portillo. Tel: +34 91 5854923. Fax: +34 915854506. e-mail: fgportillo@cnb.uam.es

1 Departamento de
Biotecnología Microbiana,
Centro Nacional de
Biotecnología, Consejo
Superior de
Investigaciones Científicas
(CSIC), Campus de
Cantoblanco, 28049
Madrid, Spain
2 Departamento de
Genética, Facultad de
Biología, Universidad de
Sevilla, 41080 Sevilla, Spain
Mutants of Salmonella enterica serovar Typhimurium lacking DNA adenine (Dam) methylase show reduced secretion of invasion effectors encoded in the Salmonella-pathogenicity island 1 (SPI-1). Concomitant with this alteration, a high number and quantity of extracellular proteins are detected in cultures of

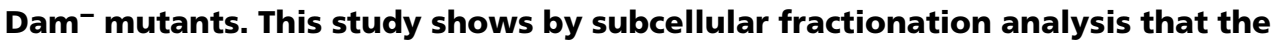
presence of numerous extracellular proteins in cultures of Dam- mutants is linked to an exacerbated release of membrane particulate material. The membrane ' leaky' phenotype and the impaired functionality of type III secretion systems were, however, unrelated since exacerbated release of proteins to the medium was evident in Dam- strains carrying mutations in either SPI-1 (invA, invJ) or flagellar (fIhD) genes. This result supports the view that Dam methylation controls a plethora of cellular processes. Electron microscopy analysis demonstrated that the accumulation of membrane particulate material occurs preferentially as vesicles in stationary cultures of Dam- $^{-}$strains. In addition, a reduction in the relative amount of peptidoglycanassociated lipoprotein (PAL), TolB, OmpA and murein lipoprotein (Lpp) bound to peptidoglycan was observed in actively growing Dam- mutants. The existence of an envelope defect was further confirmed by the increased sensitivity to deoxycholate exhibited by Dam- mutants, mostly during exponential growth. Unexpectedly, lack of Dam methylation neither increased envelope instability nor impaired the association of PAL-Tol-Lpp proteins to the peptidoglycan in Escherichia coli. Accordingly, E. coli Dam- mutants did not show sensitivity to deoxycholate. Altogether, these results indicate that, besides its role in modulating the secretion of effectors by the SPI-1-encoded type III apparatus, Dam methylation controls cell envelope integrity in $S$. enterica.

Keywords: Dam methylation, membrane vesicle, protein secretion

\section{INTRODUCTION}

Methylation of deoxyadenosine residues is found in DNAs from diverse groups of Proteobacteria (Barras \& Marinus, 1989; Marinus, 1996; Casadesús \& Torreblanca, 1996; Reisenauer et al., 1999). In Escherichia coli and Salmonella enterica, the enzyme DNA adenine methyltransferase (or Dam methylase) methylates aden-

Abbreviations: Dam, DNA adenine methylase; $\mathrm{PAL}$, peptidoglycanassociated lipoprotein; PG, peptidoglycan (fraction); SP, secreted protein; SPI-1, Salmonella-pathogenicity island 1; TP, total protein; VES, vesicle; VSN, vesicle supernatant. osine residues embedded in $5^{\prime}$-GATC- $3^{\prime}$ sites, using $S$ adenosylmethionine as the methyl donor (Lacks \& Greenberg, 1977; Hattman et al., 1978). Dam methylation is post-replicative and causes changes in DNA structure that can influence protein binding (Sternberg, 1985; Bolker \& Kahmann, 1989; Campbell \& Kleckner, 1990). The methylation state of one or more critical GATC sites can serve as a signal to modulate cellular processes such as DNA replication (Boye \& LobnerOlesen, 1990; Campbell \& Kleckner, 1990), mismatch repair (Radman \& Wagner, 1986), chromosome segregation (Ogden et al., 1988), transposition of insertion elements (Roberts et al., 1985), synthesis of fimbriae 
(Braaten et al., 1994; van der Woude \& Low, 1994; van der Woude et al., 1996; Nicholson \& Low, 2000), and bacterial conjugation (Torreblanca \& Casadesus, 1996; Torreblanca et al., 1999). A subset of these phenomena involves transcriptional gene regulation by Dam methylation (Roberts et al., 1985; Bolker \& Kahmann, 1989; van der Woude \& Low, 1994; Heithoff et al., 1999; Nicholson \& Low, 2000).

Recent studies have shown that mutants of $S$. enterica serovar Typhimurium lacking Dam methylase are highly attenuated for virulence in the murine typhoid model (Heithoff et al., 1999; Garcia-del Portillo et al., 1999; Low et al., 2001). DNA methylation is thus essential for virulence. Current evidence suggests that the role of Dam methylation in Salmonella virulence is multifactorial: Dam ${ }^{-}$mutants show defects in several virulence-related traits such as invasion of the intestinal epithelium and cytotoxicity to $\mathrm{M}$ cells (Garcia-del Portillo et al., 1999). In vitro infection assays have also shown that Dam ${ }^{-}$mutants survive within professional phagocytic cells while having reduced capacity to invade cultured non-phagocytic cells (Garcia-del Portillo et al., 1999). This partial inability to invade epithelial cells was tentatively related to reduced secretion of InvJ and SipC, two invasion proteins encoded in the Salmonellapathogenicity island 1 (SPI-1) (Garcia-del Portillo et al., 1999). Besides the impaired secretion of invasion proteins, dam mutations also cause a marked increase in the amount and number of proteins that are present in the extracellular medium (Garcia-del Portillo et al., 1999).

The spontaneous loss of membrane material by Gramnegative enteric bacteria is known to increase in the absence of a specific subset of inner-membrane, periplasmic and outer-membrane proteins that maintain envelope integrity. That is the case for the Braun's (murein) lipoprotein (Lpp); the peptidoglycan-associated lipoprotein (PAL) (Lazzaroni \& Portalier, 1992); the periplasmic protein TolB; and the inner-membrane complex formed by TolQ, TolR and TolA proteins (Lazzaroni et al., 1999). A series of studies have provided evidence for the existence of large complexes containing these proteins that establish a physical link between the inner and the outer membrane (Bouveret et al., 1995, 1999; Rigal et al., 1997; Clavel et al., 1998; Lazzaroni et al., 1999). This function is essential for providing stability and integrity to the cell envelope. Thus, mutants defective in any of these proteins share distinct phenotypic traits such as shedding of membrane vesicles, release of periplasmic proteins and hypersensitivity to detergents (Lazzaroni et al., 1999). Nevertheless, this sotermed 'membrane-leakage' phenotype has been differentiated from the spontaneous shedding of outermembrane vesicles that occurs, though to a much lesser extent, in actively growing bacteria (Kadurugamuwa \& Beveridge, 1997).

We describe below a correlation between the accumulation of proteins in the extracellular medium of $S$. enterica $\mathrm{Dam}^{-}$cultures and the disorganization of the cell envelope. Dam ${ }^{-}$mutants in exponential-growth phase are sensitive to deoxycholate and show reduced association to peptidoglycan of the envelope proteins PAL, TolB, OmpA and murein Lpp. As a result, lack of Dam methylation causes release of membrane vesicles to the extracellular medium of stationary cultures. Interestingly, these phenotypes occur concomitantly with the defect in secretion of SPI-1-encoded invasion proteins. Altogether, these data support the view that Dam methylation is necessary for both the proper activity of the SPI-1 type III secretion system and the maintenance of envelope integrity in Salmonella. We also describe that E. coli $\mathrm{Dam}^{-}$mutants do not display an equivalent phenotype of envelope instability.

\section{METHODS}

Bacterial strains and growth conditions. The Salmonella enterica and Escherichia coli strains used in this study are listed in Table 1. All S. enterica strains belong to the serovar Typhimurium and derive from the virulent strains SL1344 and 14028 s, or from the laboratory strain LT2. E. coli strains GM28 and GM33 were provided by M. G. Marinus, Department of Pharmacology, University of Massachusetts, Worcester, MA, USA. Cultures were prepared in LuriaBertani (LB) broth and incubated overnight at $37^{\circ} \mathrm{C}$ without shaking. When actively growing bacteria were required, the overnight culture was diluted 1:500 and growth was resumed to reach an $\mathrm{OD}_{550}$ of $0 \cdot 3$.

Deoxycholate sensitivity assays. Deoxycholate sensitivity was tested on LB plates containing $1 \%$ sodium deoxycholate. Bacteria were grown in LB broth, centrifuged, suspended in PBS $\mathrm{pH} 7 \cdot 4$, and diluted as appropriate in the same buffer. Drops of $0.05 \mathrm{ml}$ were then placed onto LB-deoxycholate agar, dried at room temperature, and incubated at $37^{\circ} \mathrm{C}$. Growth was monitored after 12-18 h. Assays were carried out in triplicate, using $10^{3}, 10^{4}$ and $10^{5}$ bacterial cells per drop.

Preparation of protein extracts. Bacteria grown in LB medium to exponential $\left(\mathrm{OD}_{550} 0 \cdot 3\right)$ or stationary phase $\left(\mathrm{OD}_{550} 1 \cdot 0\right)$ were spun down by centrifugation at $10000 \mathrm{~g}, 15 \mathrm{~min}, 4^{\circ} \mathrm{C}$. The supernatant was filtered in a Millipore $0 \cdot 22 \mu \mathrm{m}$ pore size filter. Proteins were precipitated in $10 \%$ trichloroacetic acid $\left(1 \mathrm{~h}, 4^{\circ} \mathrm{C}\right)$, washed in acetone, suspended in PBS pH 7.4, and boiled in SDS-loading buffer as described by Kaniga et al. (1995). These proteins constituted the 'secreted protein' (SP) fraction. The bacterial pellet was washed twice in PBS $\mathrm{pH} 7 \cdot 4$ and boiled in SDS-loading buffer to obtain the cell-associated 'total protein' (TP) fraction. To determine the presence of particulate material in the supernatant, the $0.22 \mu \mathrm{m}$ filtrate was subjected to high-speed centrifugation $\left(300000 \mathrm{~g}, 4^{\circ} \mathrm{C}\right.$, $20 \mathrm{~min}$ ) and the pellet was boiled in SDS-loading buffer. The proteins present in this pellet constituted the 'vesicle' (VES) fraction. Proteins remaining in the supernatant after highspeed centrifugation were precipitated with the $10 \%$ trichloroacetic acid/acetone procedure as described above. This fraction was named 'vesicle-supernatant' (VSN), which contained soluble extracellular proteins. All subcellular fractions were analysed for protein content by SDS-PAGE in Tris/ Tricine buffer by using $8 \%$ or $10 \%$ acrylamide gels (Shägger \& von Jagow, 1987).

Analysis of membrane and peptidoglycan-associated proteins. A subcellular fractionation method was followed to determine the relative amount of envelope proteins that were 
Table 1. Bacterial strains used in this study

\begin{tabular}{|c|c|c|}
\hline Strain & Relevant genotype & Source/reference* \\
\hline \multicolumn{3}{|c|}{ S. enterica } \\
\hline LT2 & Wild-type & Laboratory stock \\
\hline SV3000 & LT2 dam-201:: Tn10dTet & Torreblanca \& Casadesus (1996) \\
\hline $14028 \mathrm{~s}$ & Wild-type, mouse virulent & E. A. Groisman \\
\hline SV4392 & 14028s dam-201::Tn10dTet & This study \\
\hline SL1344 & hisG rpsL, mouse virulent & Hoiseth \& Stocker (1981) \\
\hline SV1610 & SL1344 dam-228::MudJ & Garcia-del Portillo et al. (1999) \\
\hline SB136 & SL1344 inv A::aphT & Jorge E. Galán \\
\hline SB302 & SL1344 invJ::aphT & Jorge E. Galán \\
\hline SV4184 & SL1344 dam-228::MudJ invA::aphT & This study \\
\hline SV4186 & SL1344 dam-228::MudJ invJ::aphT & This study \\
\hline SV4316 & SL1344 flbDC: : MudA & This study \\
\hline SV4331 & SL1344 flbDC:: MudA dam-228::MudJ & This study \\
\hline \multicolumn{3}{|l|}{ E. coli } \\
\hline GM28 & $\mathrm{F}^{-}$sup-85 (Am) & M. G. Marinus \\
\hline GM33 & $\mathrm{F}^{-}$sup-85 (Am) dam-3 & M. G. Marinus \\
\hline
\end{tabular}

*E. A. Groisman, Department of Molecular Microbiology, University of Washington School of Medicine, St Louis, MO, USA; J. E. Galan, Section of Microbial Pathogenesis, Yale School of Medicine, New Haven, CT, USA; M. G. Marinus, Department of Pharmacology, University of Massachusetts, Worcester, MA, USA.

present in inner/outer membranes or firmly associated with peptidoglycan. Thus, $\sim 10^{10}$ bacteria were spun down $\left(15000 \mathrm{~g}, 15 \mathrm{~min}, 4^{\circ} \mathrm{C}\right)$, washed and suspended in PBS $\mathrm{pH} 7 \cdot 4$ buffer. The cells were disrupted by sonication. Unbroken cells were removed by low-speed centrifugation, $5000 \mathrm{~g}, 5 \mathrm{~min}$, $4{ }^{\circ} \mathrm{C}$. The supernatant was centrifuged at high speed $\left(200000 \mathrm{~g}, 20 \mathrm{~min}, 4^{\circ} \mathrm{C}\right.$ ) to obtain a pellet containing the envelope material, which was then treated with $0.4 \%$ Triton $\mathrm{X}-100\left(3 \mathrm{~h}, 4^{\circ} \mathrm{C}\right)$ and centrifuged at $15000 \mathrm{~g}, 30 \mathrm{~min}, 4^{\circ} \mathrm{C}$ in a microfuge. The $0.4 \%$ Triton $\mathrm{X}-100$ soluble material corresponded to the 'Sol' fraction, enriched in inner-membrane proteins. The insoluble pellet was suspended in PBS $\mathrm{pH} 7.4$ and corresponded to the fraction enriched in outermembrane proteins ('Ins' fraction). Both the 'Sol' and 'Ins' fractions were finally suspended in SDS-sample buffer, boiled for $5 \mathrm{~min}$ and cleared by centrifugation before loading the samples in gels. To analyse proteins covalently bound or strongly associated with peptidoglycan, $\sim 2 \times 10^{11}$ bacteria were used to purify peptidoglycan. Bacteria were spun down and washed as described above. The pellet was then suspended in $1.5 \mathrm{ml}$ PBS $\mathrm{pH} 7.4$ buffer, and slowly mixed with $1.5 \mathrm{ml}$ boiling $8 \%$ SDS. Boiling at $100{ }^{\circ} \mathrm{C}$ was carried out for $3 \mathrm{~h}$ and then continued overnight at $80^{\circ} \mathrm{C}$. Macromolecular peptidoglycan was recovered by high-speed centrifugation $(300000 \mathrm{~g}$, $\left.20 \mathrm{~min}, 30^{\circ} \mathrm{C}\right)$. After four washing steps with warm $\left(60^{\circ} \mathrm{C}\right)$ distilled water, the pellet was suspended in $50 \mathrm{mM}$ phosphate buffer $\mathrm{pH} 4.9$ and digested overnight at $37^{\circ} \mathrm{C}$ with $\mathrm{N}, \mathrm{O}$ diacetyl-muramidase (Cellosyl, Hoescht) $\left(20 \mu \mathrm{g} \mathrm{ml}^{-1}, 37^{\circ} \mathrm{C}\right.$, $18 \mathrm{~h}$ ). Samples were incubated at $100^{\circ} \mathrm{C}$ for $20 \mathrm{~min}$ to inactivate the enzyme. Proteins released to the supernatant by peptidoglycan digestion were precipitated by simultaneous treatment with acid $\mathrm{pH}$ ( $\mathrm{pH} 4.6$ of muramidase buffer) and heat $\left(100{ }^{\circ} \mathrm{C}, 10 \mathrm{~min}\right)$, a step included in the standard procedure to prepare muropeptides for HPLC analysis (Glauner, 1988). After centrifugation at $15000 \mathrm{~g}, 15 \mathrm{~min}, 4^{\circ} \mathrm{C}$, the pellet containing the precipitated proteins and undigested peptidoglycan was suspended in $40 \mu \mathrm{l}$ PBS $\mathrm{pH} 7 \cdot 4$. After addition of SDS-sample buffer, the sample was centrifuged $\left(15000 \mathrm{~g}, 5 \mathrm{~min}, 4^{\circ} \mathrm{C}\right)$ to remove undigested macromolecular peptidoglycan. The supernatant, containing proteins firmly associated with peptidoglycan (PG fraction) was then subjected to electrophoretic protein analysis. In all cases, the sample volume to load in the gels was adjusted by the optical density value of the culture at the time at which bacteria were collected.

Western analysis and antibodies. Western analysis was performed using a Bio-Rad blotting system for protein transfer to nitrocellulose membranes. Specific proteins present in the different subcellular fractions were detected with the following primary antibodies: polyclonal rabbit anti-PAL and anti-TolB antibodies (a gift of Professor J.-C. Lazzaroni, CNRS-INSAUniversité de Lyon, France), polyclonal rabbit anti-murein lipoprotein (Lpp) (a gift from M. Inouye, Robert Wood Johnson Medical School, Piscataway, NJ, USA), affinitypurified polyclonal rabbit anti-OmpA (a gift from Heinz Schwarz, Universität Tübingen, Germany), polyclonal rabbitanti SipC from our sera collection raised against the SipC peptide ASDEARESSRKS, and polyclonal rabbit anti-ribosomal elongation factor Tu (a gift from M. Vicente, CNBCSIC, Madrid, Spain). As secondary antibody, goat antirabbit IgG conjugated to peroxidase was used for the ECLassay as described by the manufacturer (AmershamPharmacia Biotech).

Electron microscopy. The release of membrane vesicles was followed by transmission electron microscopy. Thus, the pellet obtained after high-speed centrifugation of culture supernatants (VES fraction) was fixed in $2 \%$ glutaraldehyde $/ 2 \%$ paraformaldehyde in PBS buffer $\mathrm{pH} 7.4$ (2 h, room temperature). After three washes (10 min each) in phosphate buffer, the pellets were post-fixed in $1 \%$ osmium tetroxide for $1 \mathrm{~h}$ at $4{ }^{\circ} \mathrm{C}$. After three additional washes with 
PBS buffer pH $7 \cdot 4$, samples were dehydrated with increasing concentrations of ethanol (from $50 \%$ to $100 \%$ ). Samples were then embedded in LR-White resin (London Resin Co., Reading, UK). Ultrathin sections were put onto nickel grids coated with a Formvar film, contrasted with lead citrate/ uranyl acetate and visualized at $80 \mathrm{kV}$ in a JEOL1200EX electron microscope. To visualize the integrity of bacterial envelope, the bacteria were fixed in the growth medium by adding a twofold concentrated glutaraldehyde/paraformaldehyde fixer solution. Fixation was maintained for $1 \mathrm{~h}$ at room temperature. After spinning down the bacteria, the pellet was washed twice with $0 \cdot 1 \mathrm{M}$ phosphate buffer $\mathrm{pH} 7 \cdot 4$ and processed for transmission electron microscopy as described for the pellet containing the VES fraction.

\section{RESULTS}

\section{S. enterica mutants deficient in Dam methylase show alterations in envelope stability}

The analysis of extracellular proteins present in cultures of S. enterica serovar Typhimurium $\mathrm{Dam}^{-}$mutants unveils the presence of a large number of proteins that are absent or scarce in the wild-type (Garcia-del Portillo et al., 1999). Two tentative explanations for this phenotype are as follows: (i) lack of Dam methylase might cause extensive loss of membrane and periplasmic proteins; or (ii) enhanced activity of secretion systems might exacerbate protein release. If the phenotype shown by the Dam ${ }^{-}$mutant reflects loss of membrane material, a subset of the proteins should be recovered in particulate material from the bacteria-free supernatant. To test this hypothesis, extracellular proteins present in supernatants of cultures from both the wild-type and a $\mathrm{Dam}^{-}$mutant were fractionated by high-speed centrifugation. Two fractions were obtained: particulate material recovered as a pellet (VES), and soluble proteins that remained in the supernatant (VSN). A sample consisting of the entire bulk of extracellular proteins (fraction SP) was also run for comparison. In agreement with a previous report (Garcia-del Portillo et al., 1999), the SP fraction of the $\mathrm{Dam}^{-}$strain contained a higher number of proteins than the isogenic, wild-type strain SL1344 (Fig. 1A). Dam ${ }^{-}$mutants derived from other strains of the serovar Typhimurium, such as 14028s and LT2, showed a similar complex pattern of extracellular proteins (data not shown).

Analysis of the VES fraction of the $\mathrm{Dam}^{-}$strain showed that over 15 proteins were present in the particulate material (Fig. 1A). Among these proteins, the most abundant were the flagellin subunit FliC and the outermembrane protein OmpA, as indicated by Western analysis and confirmed by controls performed with isogenic FliC $^{-}$and $\mathrm{OmpA}^{-}$null mutants (data not shown). Unlike the Dam ${ }^{-}$mutant, the particulate VES fraction of the wild-type strain contained a reduced number and amount of proteins, of which the most abundant was identified as FliC (Fig. 1A). These data suggested that lack of Dam methylase caused enhanced release of membranous material to the extracellular medium. As expected, analysis of the VSN fractions showed that the wild-type strain secretes flagellin and a
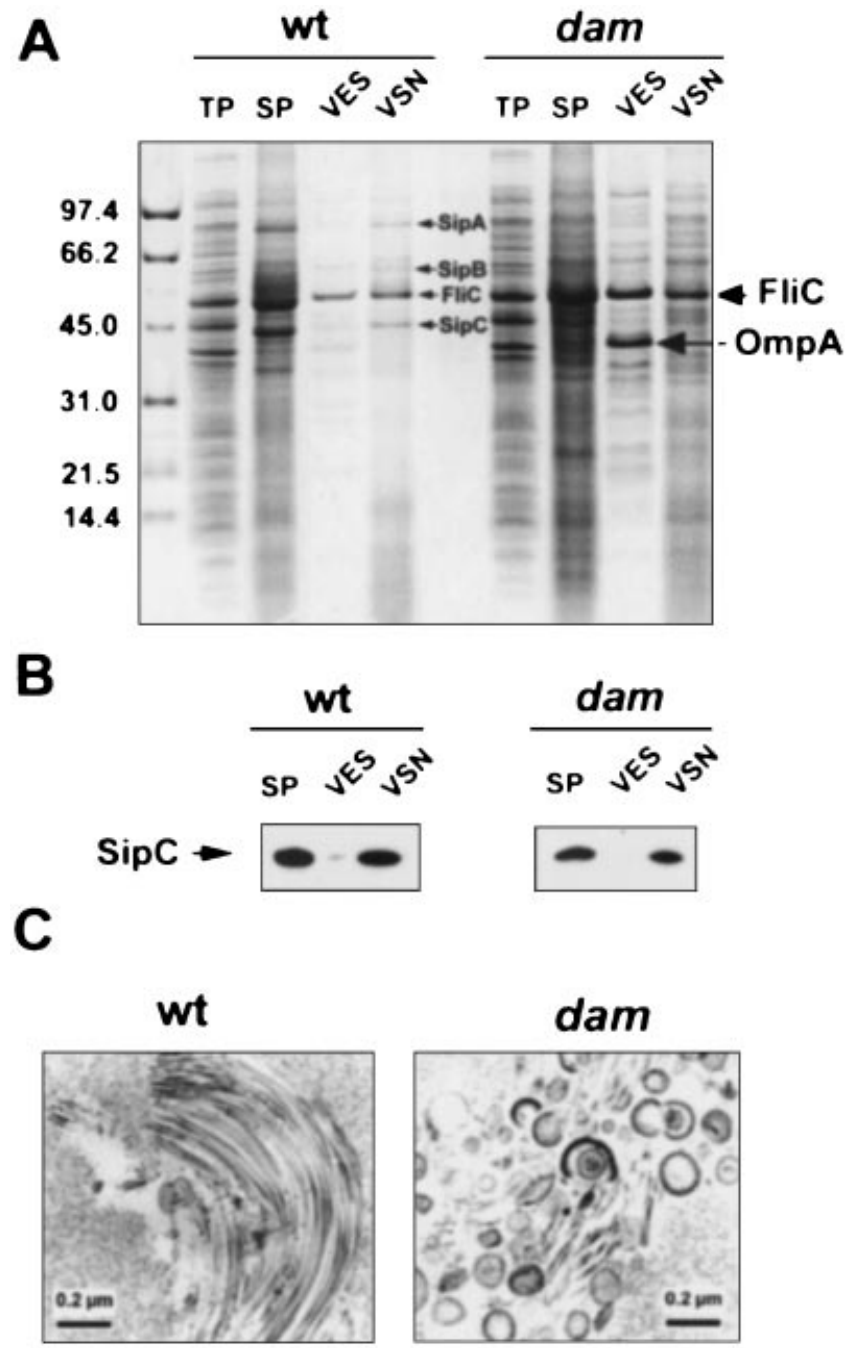

Fig. 1. Cultures of $\mathrm{Dam}^{-}$mutants of $S$. enterica contain a large number of extracellular proteins. (A) Subcellular fractions containing whole-cell-associated protein (TP), total extracellular protein (SP), extracellular protein collected by high-speed centrifugation (VES), and soluble extracellular protein (VSN), were prepared from SL1344 $\left(\mathrm{Dam}^{+}\right)$and SV1610 (Dam $\left.{ }^{-}\right)$strains at stationary phase in standing non-shaking conditions. Proteins migrating as invasion factors (SipA, SipB, SipC), flagellin FliC and outer-membrane protein (OmpA) are indicated. (B) Presence of the invasion protein SipC in extracellular fractions (SP, VES, VSN) of overnight, stationary cultures of SL1344 (Dam $\left.{ }^{+}\right)$and SV1610 (Dam $\left.{ }^{-}\right)$strains. (C) Transmission electron microscopy of particulate material (VES fraction) obtained from overnight, stationary cultures of SL1344 $\left(\mathrm{Dam}^{+}\right)$and SV1610 (Dam $\left.{ }^{-}\right)$strains. Note the presence of flagella in the sample of the wild-type (wt) strain and numerous membrane vesicles in the case of the $\mathrm{Dam}^{-}$mutant. Bars, $0.2 \mu \mathrm{m}$.

small subset of proteins that migrate in gels as the invasion-related determinants SipA, SipB and SipC (Fig. 1A) (Komoriya et al., 1999). Similarly to the VES fraction, the VSN fraction obtained from the Dam ${ }^{-}$ mutant contained a larger number of proteins (Fig. 1A), whose nature and origin are described below. 
Table 2. Effect of dam mutations on deoxycholate sensitivity

\begin{tabular}{|c|c|c|c|}
\hline \multirow[t]{2}{*}{ Strain } & \multirow{2}{*}{ Relevant genotype } & \multicolumn{2}{|c|}{ Growth on $1 \%$ deoxycholate } \\
\hline & & $\begin{array}{l}\text { Exponential } \\
\text { culture }\end{array}$ & Stationary culture \\
\hline \multicolumn{4}{|l|}{ S. enterica } \\
\hline SL1344 & $\mathrm{dam}^{+}$ & ++ & +++ \\
\hline SV1610 & dam-228::MudJ, isogenic SL1344 & - & + \\
\hline $14028 \mathrm{~s}$ & $d a m^{+}$ & +++ & +++ \\
\hline SV4392 & dam-201::MudJ, isogenic $14028 \mathrm{~s}$ & - & ++ \\
\hline LT2 & $d a m^{+}$ & ++ & +++ \\
\hline SV3000 & dam-201::Tn10dTet, isogenic LT2 & - & + \\
\hline \multicolumn{4}{|l|}{ E. coli } \\
\hline GM28 & $\mathrm{dam}^{+}$ & ++ & +++ \\
\hline GM33 & dam-3, isogenic GM28 & ++ & +++ \\
\hline
\end{tabular}

It is known that specific invasion proteins, such as the Ipa proteins of Shigella flexneri, aggregate in the extracellular medium (Parsot et al., 1995). With this observation in mind, we compared the relative amount of the invasion protein SipC in the extracellular fractions SP, VES and VSN of Dam ${ }^{+}$and Dam $^{-}$strains. Western analysis showed that the amount of SipC secreted by the $\mathrm{Dam}^{-}$mutant was lower, in the range of $50 \%$, than that of the wild-type (compare SP fractions, Fig. 1B). However, most of the SipC protein detected in the SP fraction appeared as non-particulate material (VSN fraction) in both $\mathrm{Dam}^{+}$and $\mathrm{Dam}^{-}$strains (Fig. 1B). This result suggested that $\mathrm{SipC}$ neither forms extensive aggregates nor associates with the membranous particulate material released by $\mathrm{Dam}^{-}$mutants (Fig. 1B). To further characterize the nature of the particulate material obtained upon high-speed centrifugation (VES fraction), this material was directly visualized by transmission electron microscopy. The analysis showed that intact flagella constituted the major extracellular particulate material of cultures containing the wild-type strain (Fig. 1C). This observation was consistent with the protein pattern observed in the VES fraction prepared from the wild-type strain (Fig. 1A). In contrast, the VES fraction of the $\mathrm{Dam}^{-}$mutant contained numerous membrane vesicles (Fig. 1C). These results indicated that lack of Dam methylation causes exacerbated loss of envelope material in the form of membrane vesicles, a process that is accompanied by the presence of a large number of soluble proteins in the extracellular medium.

\section{S. enterica mutants deficient in Dam methylase are sensitive to deoxycholate}

The level of resistance to deoxycholate shows variations among strains of $S$. enterica serovar Typhimurium: for instance, strain 14028s is resistant to higher deoxycholate concentrations than SL1344 and LT2. However, under the conditions chosen for our experiments (LB plates containing $1 \%$ sodium deoxycholate), strains SL1344, 14028s and LT2 were all resistant to deoxycholate, while their Dam ${ }^{-}$derivatives were sensitive (Table 2). An additional observation was that, irrespective of their parental origin, $\mathrm{Dam}^{-}$mutants were more sensitive to deoxycholate during exponential growth than in the stationary phase (Table 2). These data provide evidence that Dam $^{-}$mutants of $S$. enterica have a defect in envelope stability, mostly during active growth. In contrast to $S$. enterica, a $\mathrm{Dam}^{-}$mutant of E. coli did not show deoxycholate sensitivity, even during exponential growth (Table 2).

\section{Loss of membrane material in S. enterica Dam- mutants occurs regardless of the activity of type III secretion systems}

Specialized type III secretion systems, such as that involved in flagellar synthesis and the SPI-1-encoded system, secrete most of the proteins that are detected in supernatants of $S$. enterica cultures (Komoriya et al., 1999). To determine whether any of the proteins present in the VSN fraction of $\mathrm{Dam}^{-}$mutants were secreted by any of these two type III systems, a dam mutation was combined with mutations in the SPI-1 genes inv $A$ and invJ (strains SV4184 and SV4186, respectively), or in the gene encoding the flagellar master regulator $f l h D$ (strain SV4331). Previous studies have shown that absence of either the inner-membrane protein InvA or the translocator InvJ render $S$. enterica unable to secrete any invasion-related protein effector (Collazo et al., 1995; Collazo \& Galán, 1997). In turn, the flagellar master regulator FlhDC is absolutely required for the synthesis of structural or secreted flagellar proteins (Komoriya et al., 1999). Protein analysis of SP, VES and VSN fractions from the single and double mutants indicated that release of particulate membrane material to the extracellular medium occurs whenever a dam mutation is present, irrespective of the functional status of the two type III secretion system analysed (SP and VES fractions 

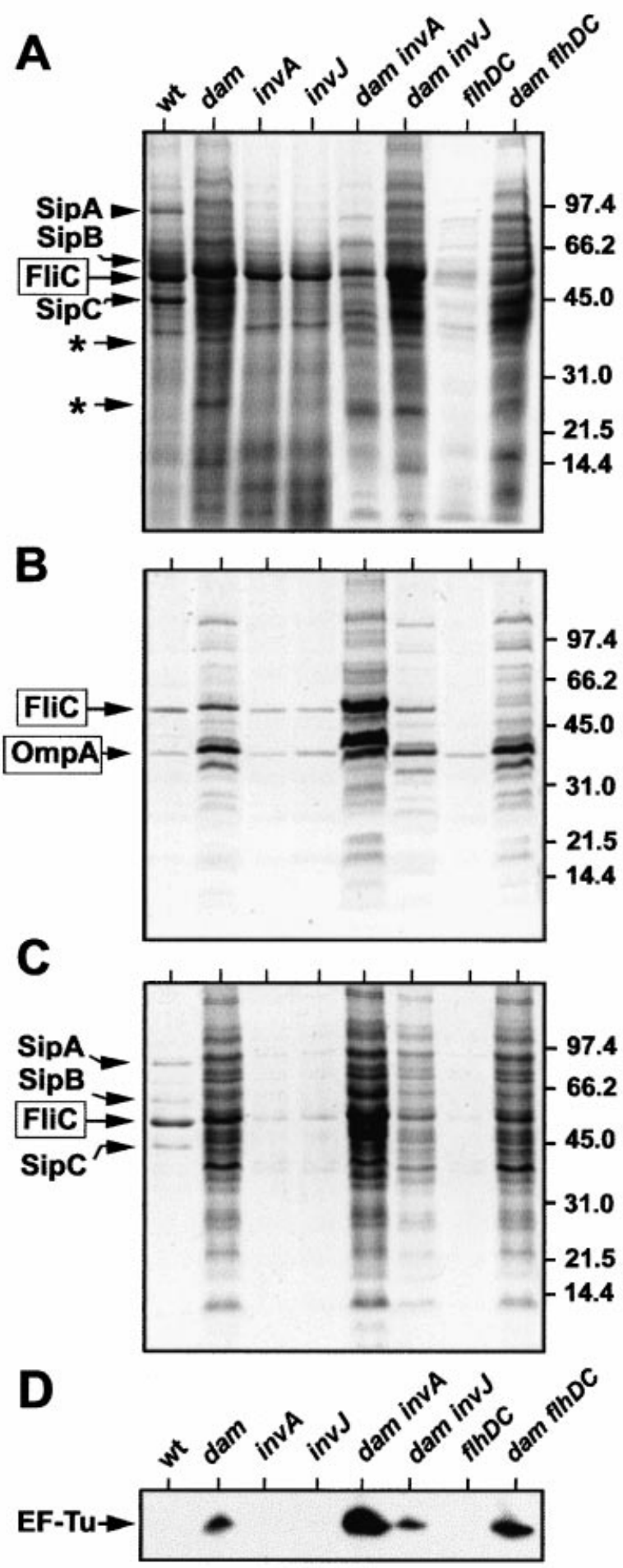

Fig. 2. Release of membrane vesicles caused by a dam mutation occurs independently of the activity of type III protein secretion systems. (A) Extracts containing total extracellular protein (SP fraction) were prepared from overnight stationary cultures of strains SL1344 (Dam $\left.{ }^{+}\right)$, SV1610 $\left(\mathrm{Dam}^{-}\right)$, SB136 (InvA $\left.)^{-}\right)$, SB302 $\left(\right.$ InvJ $\left.^{-}\right)$, SV4184 (Dam ${ }^{-}$InvA $\left.^{-}\right)$, SV4186 $\left(\right.$Dam $^{-}$InvJ $\left.^{-}\right)$, SV4316 $\left(\mathrm{FlhDC}^{-}\right)$and SV4331 (Dam FlhDC $^{-}$). The Sips and FliC proteins are indicated. The asterisks mark two proteins only visible in extracts of strains carrying the dam mutation. (B) Extracts containing extracellular particulate material (VES fraction) of the same set of strains detailed in (A). The two major proteins shown in Fig. 2A, B, respectively). Moreover, most of the soluble extracellular proteins that appear in the VSN fraction of the single Dam ${ }^{-}$mutant were still present in VSN fractions from double mutants lacking functional SPI-1 or flagellar type III systems (Fig. 2C). These results raised the question of whether the proteins present in the VSN fraction could be of cytosolic origin, thereby reflecting bacterial lysis. This hypothesis was tested by Western analysis using an antibody against the ribosomal elongation factor Tu, EF-Tu. The results shown in Fig. 2(D) demonstrated that bacterial lysis occurred in cultures of all strains harbouring a dam mutation. Lysis affected $2-5 \%$ of the bacterial population, as estimated by comparing the levels of EF-Tu in the extracellular extract versus the levels present in whole-cell-associated extracts (data not shown). Hence, lack of Dam methylation causes loss of membrane and cytosolic material associated with the loss of viability in a small proportion of the bacterial population.

\section{Lack of Dam methylase causes loss of membrane particulate material in stationary-phase bacteria}

Shedding of membrane vesicles is known to occur in many Gram-negative bacteria during the active phase of growth (Whitmire \& Garon, 1993; Kadurugamuwa \& Beveridge, 1995, 1997; Wai et al., 1995; Zhou et al., 1998). Electron microscopy analysis of the VES fraction unveiled the existence of membrane vesicles in the extracellular medium of saturated, stationary cultures of Dam ${ }^{-}$mutants (see Fig. 1C). To determine whether this loss of membrane material due to lack of Dam methylase occurred during exponential or stationary growth, the morphology of the bacterial envelope was examined by electron microscopy. These experiments failed to demonstrate visible differences in the envelope along the surface of wild-type and $\mathrm{Dam}^{-}$bacteria (Fig. $3 \mathrm{~A}-\mathrm{D})$. Lack of major alterations in envelope integrity was noticed in both exponential and stationary cultures (Fig. 3A-D). However, the appearance of membranous material and vesicles was consistently reproduced in the samples obtained from stationary-phase cultures of Dam $^{-}$mutants (Fig. 3E). This result indicated that accumulation of membrane particulate material only occurs in stationary cultures of $\mathrm{Dam}^{-}$mutants. The possibility that vesicle release starts during exponential growth and increases swiftly cannot be excluded. However, our failure to detect membrane vesiculation in actively growing bacteria is more consistent with the view that loss of membrane material starts when the Dam $^{-}$mutant enters the stationary phase.

present that correspond to FliC and OmpA are indicated. (C) Extracts containing extracellular soluble material (VSN fraction) of the same set of strains detailed in (A). The Sips and FliC proteins are indicated. (D) Immuno-detection of the cytosolic elongation ribosomal factor EF-Tu in the fractions containing soluble proteins (VSN fractions) shown in (C). Note that the strains that release this cytosolic protein are those harbouring the dam mutation. The percentage of lysis was estimated as $2-5 \%$ upon comparison of this signal with that obtained from whole-cell protein extracts (not shown). wt, wild-type. 


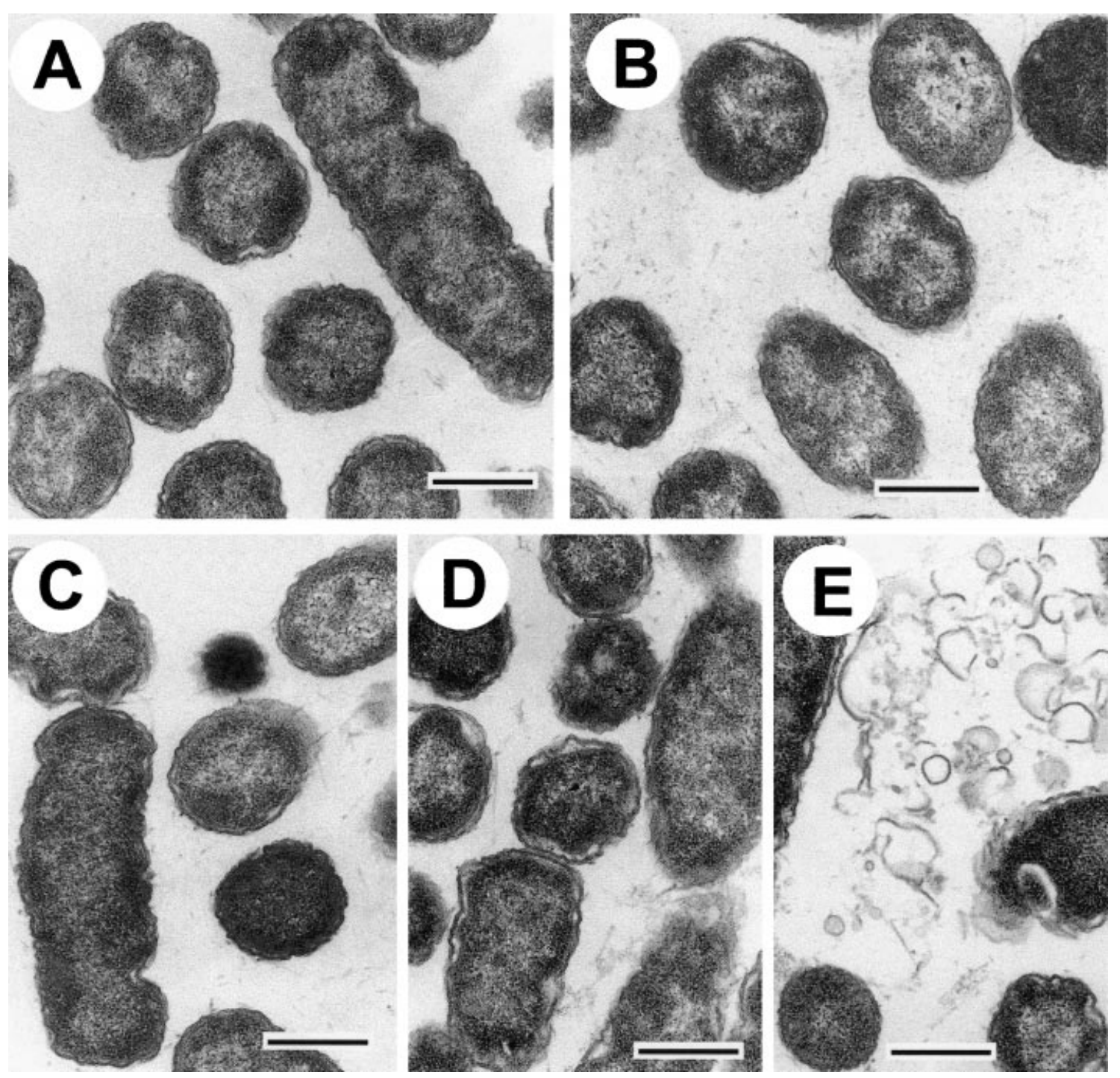

Fig. 3. S. enterica Dam $^{-}$mutants do not display massive envelope alterations. Transmission electron microscopy of bacteria grown to exponential phase $\left(\mathrm{OD}_{550} 0 \cdot 3\right)(\mathrm{A}, \mathrm{B})$ or to stationary phase (C-E). (A, C) SL1344 (Dam $\left.{ }^{+}\right) ;(B, D, E) S V 1610$ $\left(\mathrm{Dam}^{-}\right)$. Note the absence of differences in the morphology of the cell envelope in wild-type and mutant bacteria grown to exponential or stationary phase. (E) Shows particulate material and vesicle membranes that were consistently observed in samples prepared from stationary phase cultures of the Dam ${ }^{-}$mutant. Bars, $0.5 \mu \mathrm{m}$.

\section{Dam methylation controls the association of PAL, TolB, Lpp and OmpA proteins with peptidoglycan}

The observation that lack of Dam methylase leads to loss of particulate material and cell viability raised the question of whether this phenotype might be triggered by a functional defect in proteins that play a role in the preservation of membrane stability. Among these proteins are the TolQRAB-PAL group and the murein (Braun's) lipoprotein (Lpp). All these proteins bind strongly, even covalently (e.g. Lpp), to the peptidoglycan layer. Lack of any of these proteins triggers extensive membrane leakage (Bernadac et al., 1998; Lazzaroni et al., 1999). To analyse a putative role of Dam methylase in the synthesis or distribution of these proteins in the bacterial cell envelope, a protocol was optimized to obtain a highly purified peptidoglycan (PG) fraction (see Methods). The relative proportion of TolB, PAL and Lpp proteins that remained firmly associated with purified peptidoglycan was tested by Western analysis. OmpA was also included in this analysis, since it is one of the major proteins present in the outer membrane and it has been reported to associate with peptidoglycan (Koebnik, 1995). PG fractions were prepared from the wild-type and a $\mathrm{Dam}^{-}$mutant in exponential and stationary phase (Fig. 4). Two additional fractions containing the protein present in the membrane, but not firmly associated with peptidoglycan (i.e. solubilized with either $0.4 \%$ Triton X-100 or $1 \%$ SDS, fractions 'Sol' and 'Insol' respectively), were also included for comparison (Fig. 4). Unlike the results obtained in stationary phase, a substantial decrease in the amount of 


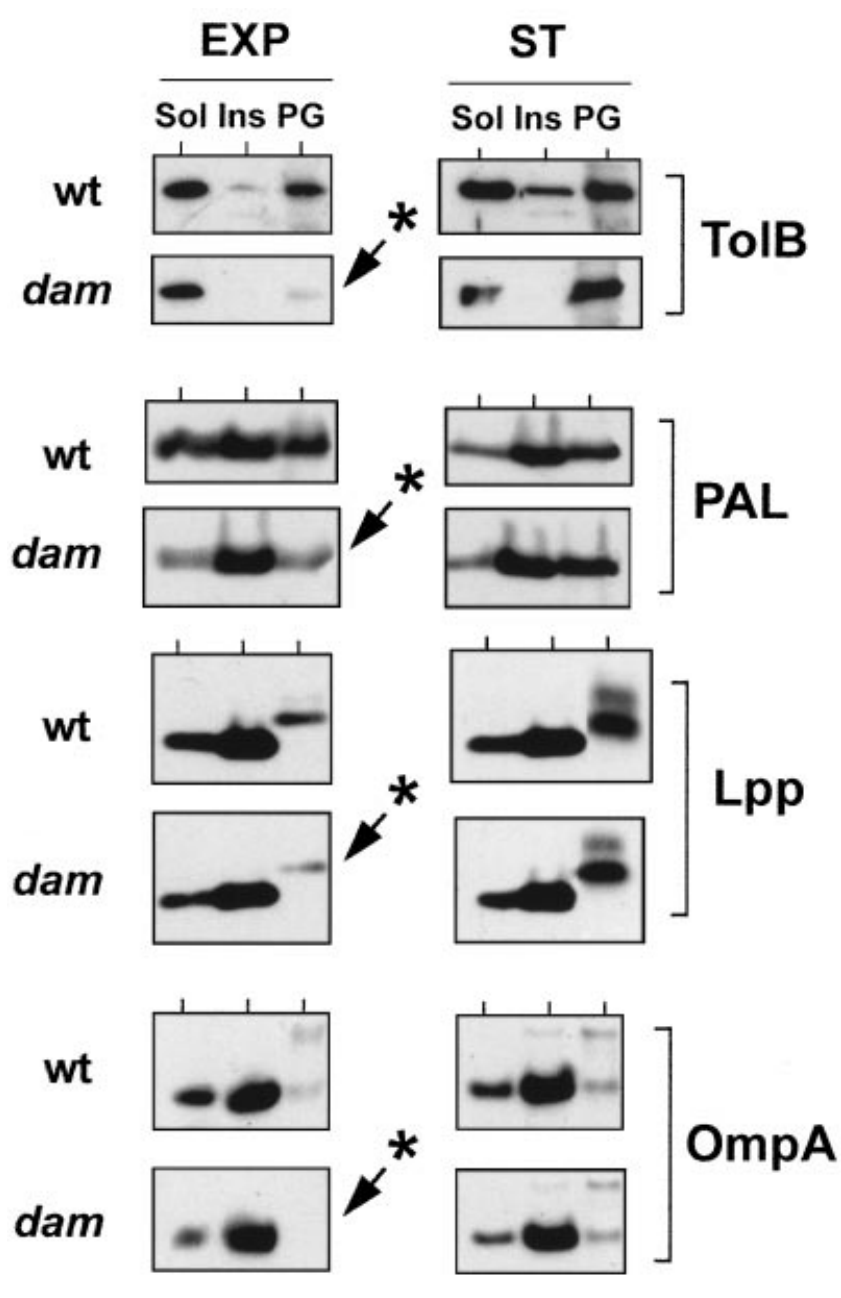

Fig. 4. Western blot analysis of the envelope distribution of TolB, PAL, Lpp and OmpA proteins in Dam $^{-}$mutants of $S$. enterica. Envelope extracts were prepared from bacteria grown to exponential phase (EXP) or stationary phase (ST). The different fractions consisted of: Sol, envelope proteins soluble in $0.4 \%$ Triton X-100; Ins, envelope proteins insoluble in $0.4 \%$ Triton X-100; PG, proteins that remain associated with peptidoglycan after extensive boiling in $4 \%$ SDS (see Methods). TolB, PAL, Lpp and OmpA proteins were detected with specific antibodies. The asterisks mark those cases in which a noticeable difference was observed. wt, wild-type.

TolB, PAL, Lpp and OmpA that associate with peptidoglycan was observed in the actively growing $\mathrm{Dam}^{-}$ mutant (Fig. 4). These differences were more marked for the TolB and PAL proteins (Fig. 4). To confirm that these differences were not related to loss of membrane material by the Dam ${ }^{-}$mutant, the relative amount of a cytosolic protein, the ribosomal factor EF-Tu, was monitored in cytosolic fractions prepared from the same number of bacteria used to prepare the envelope fractions. In this case, an equivalent amount of EF-Tu was observed for both the wild-type and the Dam ${ }^{-}$ mutant (data not shown). Altogether, these results suggest that Dam methylation plays a relevant role in controlling the distribution and relative amount of

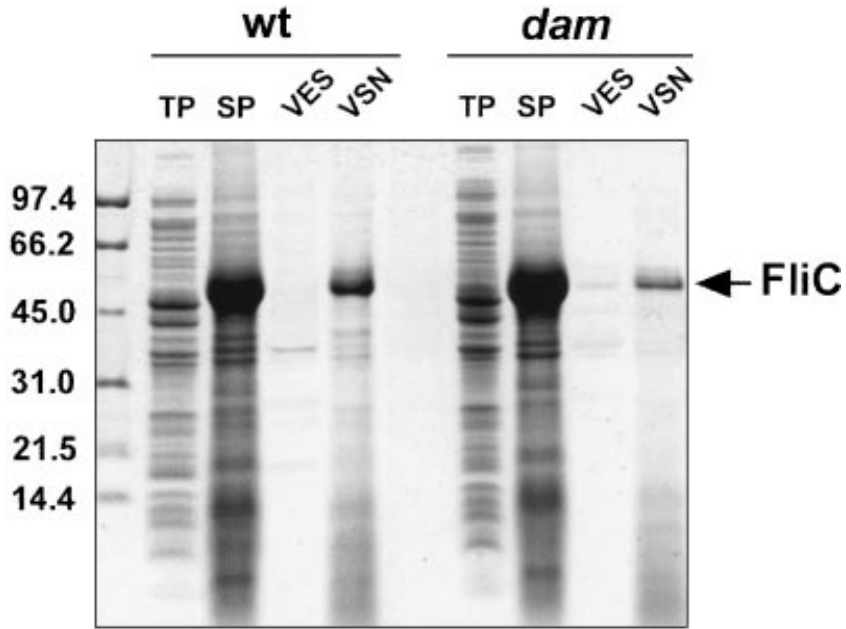

Fig. 5. E. coli $\mathrm{Dam}^{-}$mutants do not exhibit a phenotype of membrane instability. Subcellular fractions TP, SP, VES and VSN (see legend to Fig. 1) were prepared from isogenic E. coli strains GM28 (Dam ${ }^{+}$) and GM33 (Dam ${ }^{-}$) grown to stationary phase. Unlike $S$. enterica, no accumulation of proteins in the extracellular medium was observed. wt, wild-type.

peptidoglycan-associated proteins when bacteria undergo active growth. This regulation is apparently exerted to a higher degree in the case of the envelope-stabilizing proteins TolB and PAL.

\section{Dam methylation is not required for maintenance of envelope integrity in $E$. coli}

S. enterica and E. coli are closely related genera. Most of the 'membrane-leakage' phenotypes associated with loss of function in Tol-PAL or Lpp proteins have been demonstrated in E. coli (Bernadac et al., 1998). Therefore, we were interested to test whether Dam methylase also played a role in the control of membrane integrity in E. coli. A fraction of total extracellular protein present in the culture supernatant (fraction SP) was prepared from isogenic E. coli wild-type and $\mathrm{Dam}^{-}$strains. Unlike S. enterica, lack of Dam methylase in E. coli did not produce massive accumulation of proteins into the extracellular medium (Fig. 5). Furthermore, when the extracellular proteins were separated by high-speed centrifugation in the corresponding particulate and soluble fractions, the VES fraction proved to have a low protein content (Fig. 5). In the VSN fraction (soluble extracellular fraction), a major protein migrating as flagellin was observed (Fig. 5). In contrast to S. enterica, no major differences were observed in the amount or distribution of TolB, PAL, OmpA and Lpp proteins between wild-type and $\mathrm{Dam}^{-}$E. coli strains during exponential or stationary growth phases (data not shown). A concomitant observation is that dam mutations do not cause deoxycholate sensitivity in E. coli (Table 2). These observations indicate that Dam methylation is not required for envelope stability in E. coli. 


\section{DISCUSSION}

In a previous study we showed that Dam methylase mutants of $S$. enterica secrete high amounts of proteins into the culture medium (Garcia-del Portillo et al., 1999). Complementation with a plasmid-borne dam gene restored the wild-type pattern of protein secretion, indicating that protein hypersecretion was solely caused by the dam mutation (Garcia-del Portillo et al., 1999). This phenotype, first described for $\mathrm{Dam}^{-}$mutants of SL1344, has been now reproduced in $\mathrm{Dam}^{-}$derivatives of other strains of $S$. enterica serovar Typhimurium, such as the laboratory strain LT2 and the mousevirulent strain 14028 s.

In an attempt to characterize the basis for this phenotype, we separated the extracellular proteins by highspeed centrifugation into two fractions: (i) particulate material; and (ii) soluble material. In the Dam ${ }^{-}$mutant, over 15 proteins were visible in the particulate material. Some of these proteins were identified as flagellin (FliC), the outer-membrane proteins OmpA, OmpC, OmpX, and the murein lipoprotein Lpp by Western blotting and mass spectrometry analysis (data not shown). This result provided us with the first evidence for an involvement of Dam methylation in the maintenance of envelope integrity. In addition, electron microscopy analysis of fractions containing particulate material showed that stationary cultures of $\mathrm{Dam}^{-}$mutants contained large amount of membrane vesicles in the extracellular medium, whereas only flagella were visible in extracts from the wild-type strain. Interestingly, direct examination of whole bacteria in either exponential or stationary growth phases failed to show massive envelope defects in the Dam ${ }^{-}$mutant. Nevertheless, and in agreement with a recent study (Heithoff et al., 2001), our results show that $\mathrm{Dam}^{-}$mutants are highly sensitive to deoxycholate during active growth. The apparent contradiction between this sensitivity and the inability to observe major defects in actively growing $\mathrm{Dam}^{-}$mutants might indicate the existence of minor alterations in envelope stability sufficient to promote sensitivity to bile acids. These envelope alterations might be cumulative and, upon entrance into the stationary phase, culminate in envelope breakage in a subpopulation of bacteria.

The existence of an envelope defect in exponential cultures of $\mathrm{Dam}^{-}$mutants was confirmed by subcellular fractionation analysis: a set of proteins required for the maintenance of envelope stability were found to associate at lowered levels with the peptidoglycan of Dam $^{-}$mutants. Among these proteins, a marked alteration of the association of TolB and PAL to peptidoglycan was observed in mutants lacking Dam methylase. Previous studies have shown that deficiency of any of these proteins in E. coli triggers release and leakage of membrane and periplasmic material into the growth medium (Bernadac et al., 1998; Lazzaroni et al., 1999). Our study provides the first evidence for a similar envelope-stabilizing role of these proteins in S. enterica and, in addition, raises the hypothesis that such a role is controlled by Dam methylation. The fact that the alterations in protein-peptidoglycan association were noticeable only in actively growing bacteria indicates that the envelope defect caused by lack of Dam methylation might reflect up-regulation of a natural process that occurs under conditions of high growth rate and cell wall turnover. This assumption agrees with previous studies that demonstrated the shedding of membrane vesicles as a process occurring predominantly in actively growing bacteria (Kadurugamuwa \& Beveridge, 1995, 1997; Zhou et al., 1998). Interestingly, and unlike Lpp and OmpA, lack of Dam methylation seems to affect the total amount of TolB and PAL proteins present in the envelope of actively growing bacteria. This observation contrasts with the similar levels of mRNA transcripts of the tolQRAB and pal operons detected in $\mathrm{Dam}^{+}$and $\mathrm{Dam}^{-}$strains (unpublished observations), which suggests the existence of post-transcriptional regulatory mechanisms acting on TolB and PAL, and controlled by Dam methylation. These post-transcriptional regulatory mechanisms could be sustained by the stimulation of a normal physiological process (i.e. cell wall turnover). Alternatively, Dam methylation might control the synthesis of an as yet unknown cell function required for proper maintenance or positioning of the Tol-PAL protein complexes. The fact that the association to peptidoglycan of all the envelope proteins analysed was altered at some degree by the lack of Dam methylase suggests that a basic process of envelope physiology may be altered.

In addition to the set of proteins present in membranous material, about 25-30 proteins were visible by Coomassie staining in the soluble, extracellular fraction (VSN) from $\mathrm{Dam}^{-}$mutants. Although we first considered that hypersecretion of VSN proteins could reflect some imbalance in protein secretion systems, two observations revealed that this was not the case. First, with the exception of the flagellin subunit FliC, the pattern of proteins present in the VSN fraction was unaltered when either the flagellar or invasion-specific type III systems were non-functional. Because most proteins secreted by $S$. enterica are either flagellar proteins or virulence-related proteins (Komoriya et al., 1999), we reasoned that many proteins present in the VSN fraction could derive from lysed bacteria. Furthermore, the detection of a ribosomal factor in the VSN fraction confirmed that the $\mathrm{Dam}^{-}$mutant undergoes lysis upon entering stationary phase. Some $2-5 \%$ of the $\mathrm{Dam}^{-}$bacterial population was estimated to lyse in stationary phase. Despite this small percentage, the concentration of extracellular proteins by acid precipitation hampered the visual analysis of 'secreted' proteins. An example was the SipC protein, which is secreted at a $50 \%$ rate in a $\mathrm{Dam}^{-}$mutant (Fig. 1B) (Garcia-del Portillo et al., 1999), but was virtually masked by the bulk of proteins present in the extracellular fraction (SP) of the Dam ${ }^{-}$mutant.

Release of outer-membrane vesicles is a physiological process that occurs naturally in many bacterial pathogens. For instance, Shigella flexneri, Pseudomonas aeruginosa, Actinobacillus pleuropneumoniae, entero- 
haemorrhagic E. coli (EHEC) and Helicobacter pylori are all known to shed membrane vesicles into the culture medium (Kadurugamuwa \& Beveridge, 1995, 1997, 1999; Wai et al., 1995; Keenan et al., 2000). In some cases, these vesicles have been related to the transport of pathogenic factors. Examples include the VacA toxin of H. pylori (Keenan et al., 2000), Shiga toxin and lipopolysaccharide by STEC (Shiga-toxin producing E. coli) and EHEC (Wai et al., 1995; Meno et al., 2000; Yokoyama et al., 2000). In other cases outermembrane vesicles have been shown to contain muramidases and other periplasmic enzymes (Kadurugamuwa \& Beveridge, 1995, 1999). When these membrane vesicles fuse with the membrane of a different bacterial species, their content can trigger loss of viability of the recipient strain, or a physiological 'incorporation' of this heterologous material into its envelope structure (Li et al., 1998; Kadurugamuwa \& Beveridge, 1999). If outer-membrane shedding is a normal physiological process in S. enterica, it seems reasonable that it must be subjected to tight control. Our study suggests that Dam methylation may participate in such a control.

Unlike S. enterica, lack of Dam methylation did not cause any noticeable effect on envelope integrity or stability in E. coli. Biochemical analyses of protein content in extracellular fractions, deoxycholate sensitivity experiments, and studies of protein-peptidoglycan interactions were all unable to show any difference between $\mathrm{Dam}^{+}$and $\mathrm{Dam}^{-}$strains of E. coli. The basis of this unexpected difference between Salmonella and $E$. coli is at present unknown. One may, however, recall that the $S$. enterica genome contains genes with a putative role in peptidoglycan remodelling which are absent in E. coli (Hilbert et al., 1999).

Envelope instability might contribute to virulence attenuation of $S$. enterica $\mathrm{Dam}^{-}$mutants in the mouse typhoid model. Enhanced release of membrane vesicles through infected cells and tissues might cause overstimulation of the host immune response, as shown for Neisseria meningitidis (Mirlashari et al., 2001). Release of outer-membrane proteins such as OmpA, PAL and Lpp to the extracellular medium can occur in the presence of serum (Hellman et al., 2000a, b), and this process has been proposed as a major phenomenon mediating elicitation of immune host defence. Therefore, overstimulation of host defences during infection might render $\mathrm{Dam}^{-}$mutants unable to cause disease. These effects on the host would also sustain the vaccine properties of S. enterica Dam ${ }^{-}$mutants (Garcia-del Portillo et al., 1999; Heithoff et al., 1999, 2001).

\section{ACKNOWLEDGEMENTS}

We are grateful to Drs J.-C. Lazzaroni, H. Schwarz, M. Inouye and M. Vicente for providing antibodies, and to Drs M. G. Marinus, J. E. Galán and E. A. Groisman for sending bacterial strains. Strain construction was carried out by Dr David Cano. This work was supported by grants from the European Union (QLK2-1999-00310) and the Comunidad de Madrid $(08.2 / 0045.1 / 2000)$. M.G.P. is supported by a postdoctoral fellowship from the Comunidad de Madrid. A.I.P. is the recipient of a predoctoral fellowship from the Fundación Ramón Areces.

\section{REFERENCES}

Barras, F. \& Marinus, M. G. (1989). The great GATC: DNA methylation in E. coli. Trends Genet 5, 139-143.

Bernadac, A., Gavioli, M., Lazzaroni, J. C., Raina, S. \& Lloubes, R. (1998). Escherichia coli tol-pal mutants form outer membrane vesicles. J Bacteriol 180, 4872-4878.

Bolker, M. \& Kahmann, R. (1989). The Escherichia coli regulatory protein OxyR discriminates between methylated and unmethylated states of the phage $\mathrm{Mu}$ mom promoter. EMBO J 8, 2403-2410.

Bouveret, E., Derouiche, R., Rigal, A., Lloubes, R., Lazdunski, C. \& Benedetti, H. (1995). Peptidoglycan-associated lipoprotein-TolB interaction. A possible key to explaining the formation of contact sites between the inner and outer membranes of Escherichia coli. J Biol Chem 270, 11071-11077.

Bouveret, E., Benedetti, H., Rigal, A., Loret, E. \& Lazdunski, C. (1999). In vitro characterization of peptidoglycan-associated lipoprotein (PAL)-peptidoglycan and PAL-TolB interactions. $J$ Bacteriol 181, 6306-6311.

Boye, E. \& Lobner-Olesen, A. (1990). The role of dam methyltransferase in the control of DNA replication in E. coli. Cell 62, 981-989.

Braaten, B. A., Nou, X., Kaltenbach, L. S. \& Low, D. A. (1994). Methylation patterns in pap regulatory DNA control pyelonephritis-associated pili phase variation in E. coli. Cell 76, 577-588.

Campbell, J. L. \& Kleckner, N. (1990). E. coli oriC and the $d n a A$ gene promoter are sequestered from Dam methyltransferase following the passage of the chromosomal replication fork. Cell 62, 967-979.

Casadesús, J. \& Torreblanca, J. (1996). Methylation-related epigenetic signals in bacterial DNA. In Epigenetic Mechanisms of Gene Regulation, pp. 141-153. Edited by V. E. A. Russo, R. A. Martienssen \& A. D. Riggs. Cold Spring Harbor, NY: Cold Spring Harbor Laboratory.

Clavel, T., Germon, P., Vianney, A., Portalier, R. \& Lazzaroni, J. C. (1998). TolB protein of Escherichia coli K-12 interacts with the outer membrane peptidoglycan-associated proteins Pal, Lpp and OmpA. Mol Microbiol 29, 359-367.

Collazo, C. M. \& Galán, J. E. (1997). The invasion-associated type III system of Salmonella typhimurium directs the translocation of Sip proteins into the host cell. Mol Microbiol 24, 747-756.

Collazo, C. M., Zierler, M. K. \& Galán, J. E. (1995). Functional analysis of the Salmonella typhimurium invasion genes invl and invJ and identification of a target of the protein secretion apparatus encoded in the inv locus. Mol Microbiol 15, 25-38.

Garcia-del Portillo, F., Pucciarelli, M. G. \& Casadesus, J. (1999). DNA adenine methylase mutants of Salmonella typhimurium show defects in protein secretion, cell invasion, and $M$ cell cytotoxicity. Proc Natl Acad Sci U S A 96, 11578-11583.

Glauner, B. (1988). Separation and quantification of muropeptides with high-performance liquid chromatography. Anal Biochem 172, 451-464.

Hattman, S., Brooks, J. E. \& Masurekar, M. (1978). Sequence specificity of the P1 modification methylase (M.Eco P1) and the DNA methylase (M.Eco dam) controlled by the Escherichia coli dam gene. J Mol Biol 126, 367-380.

Heithoff, D. M., Sinsheimer, R. L., Low, D. A. \& Mahan, M. J. 
(1999). An essential role for DNA adenine methylation in bacterial virulence. Science 284, 967-970.

Heithoff, D. M., Enioutina, E. Y., Daynes, R. A., Sinsheimer, R. L., Low, D. A. \& Mahan, M. J. (2001). Salmonella DNA adenine methylase mutants confer cross-protective immunity. Infect Immun 69, 6725-6730.

Hellman, J., Loiselle, P. M., Tehan, M. M., Allaire, J. E., Boyle, L. A., Kurnick, J. T., Andrews, D. M., Sik Kim, K. \& Warren, H. S. (2000a). Outer membrane protein A, peptidoglycan-associated lipoprotein, and murein lipoprotein are released by Escherichia coli bacteria into serum. Infect Immun 68, 2566-2572.

Hellman, J., Loiselle, P. M., Zanzot, E. M., Allaire, J. E., Tehan, M. M., Boyle, L. A., Kurnick, J. T. \& Warren, H. S. (2000b). Release of gram-negative outer-membrane proteins into human serum and septic rat blood and their interactions with immunoglobulin in antiserum to Escherichia coli J5. J Infect Dis 181, 1034-1043.

Hilbert, F., Garcia-del Portillo, F. \& Groisman, E. A. (1999). A periplasmic D-alanyl-D-alanine dipeptidase in the gram-negative bacterium Salmonella enterica. J Bacteriol 181, 2158-2165.

Hoiseth, S. K. \& Stocker, B. A. (1981). Aromatic-dependent Salmonella typhimurium are non-virulent and effective as live vaccines. Nature 291, 238-239.

Kadurugamuwa, J. L. \& Beveridge, T. J. (1995). Virulence factors are released from Pseudomonas aeruginosa in association with membrane vesicles during normal growth and exposure to gentamicin: a novel mechanism of enzyme secretion. J Bacteriol 177, 3998-4008.

Kadurugamuwa, J. L. \& Beveridge, T. J. (1997). Natural release of virulence factors in membrane vesicles by Pseudomonas aeruginosa and the effect of aminoglycoside antibiotics on their release. J Antimicrob Chemother 40, 615-621.

Kadurugamuwa, J. L. \& Beveridge, T. J. (1999). Membrane vesicles derived from Pseudomonas aeruginosa and Shigella flexneri can be integrated into the surfaces of other gram-negative bacteria. Microbiology 145, 2051-2060.

Kaniga, K., Trollinger, D. \& Galán, J. E. (1995). Identification of two targets of the type III protein secretion system encoded by the inv and spa loci of Salmonella typhimurium that have homology to the Shigella IpaD and IpaA proteins. J Bacteriol 177, 7078-7085.

Keenan, J., Day, T., Neal, S., Cook, B., Perez-Perez, G., Allardyce, R. \& Bagshaw, P. (2000). A role for the bacterial outer membrane in the pathogenesis of Helicobacter pylori infection. FEMS Microbiol Lett 182, 259-264.

Koebnik, R. (1995). Proposal for a peptidoglycan-associating alpha-helical motif in the C-terminal regions of some bacterial cell-surface proteins. Mol Microbiol 16, 1269-1270.

Komoriya, K., Shibano, N., Higano, T., Azuma, N., Yamaguchi, S. \& Aizawa, S. I. (1999). Flagellar proteins and type III-exported virulence factors are the predominant proteins secreted into the culture media of Salmonella typhimurium. Mol Microbiol 34, 767-779.

Lacks, S. \& Greenberg, B. (1977). Complementary specificity of restriction endonucleases of Diplococcus pneumoniae with respect to DNA methylation. J Mol Biol 114, 153-168.

Lazzaroni, J. C. \& Portalier, R. (1992). The excC gene of Escherichia coli $\mathrm{K}-12$ required for cell envelope integrity encodes the peptidoglycan-associated lipoprotein (PAL). Mol Microbiol 6, 735-742.

Lazzaroni, J. C., Germon, P., Ray, M. C. \& Vianney, A. (1999). The Tol proteins of Escherichia coli and their involvement in the uptake of biomolecules and outer membrane stability. FEMS Microbiol Lett 177, 191-197.
Li, Z., Clarke, A. J. \& Beveridge, T. J. (1998). Gram-negative bacteria produce membrane vesicles which are capable of killing other bacteria. J Bacteriol 180, 5478-5483.

Low, D. A., Weyand, N. J. \& Mahan, M. J. (2001). Roles of DNA adenine methylation in regulating bacterial gene expression and virulence. Infect Immun 69, 7197-7204.

Marinus, M. G. (1996). Methylation of DNA. In Escherichia coli and Salmonella: Cellular and Molecular Biology, 2nd edn, pp. 782-791. Edited by F. C. Neidhardt and others. Washington, DC: American Society for Microbiology.

Meno, Y., Fujimoto, S., Horikawa, K. \& Yoshida, S. (2000). Release of membrane vesicles containing endotoxic lipopolysaccharide in Escherichia coli O157:H7 clinical isolates. Microbiol Immunol 44, 271-274.

Mirlashari, M. R., Hoiby, E. A., Holst, J. \& Lyberg, T. (2001). Outer membrane vesicles from Neisseria meningitidis: effects on cytokine production in human whole blood. Cytokine 13, 91-97.

Nicholson, B. \& Low, D. (2000). DNA methylation-dependent regulation of pef expression in Salmonella typhimurium. Mol Microbiol 35, 728-742.

Ogden, G. B., Pratt, M. J. \& Schaechter, M. (1988). The replicative origin of the E. coli chromosome binds to cell membranes only when hemimethylated. Cell 54, 127-135.

Parsot, C., Menard, R., Gounon, P. \& Sansonetti, P. J. (1995). Enhanced secretion through the Shigella flexneri Mxi-Spa translocon leads to assembly of extracellular proteins into macromolecular structures. Mol Microbiol 16, 291-300.

Radman, M. \& Wagner, R. (1986). Mismatch repair in Escherichia coli. Annu Rev Genet 20, 523-538.

Reisenauer, A., Kahng, L. S., McCollum, S. \& Shapiro, L. (1999). Bacterial DNA methylation: a cell cycle regulator? J Bacteriol 181, 5135-5139.

Rigal, A., Bouveret, E., Lloubes, R., Lazdunski, C. \& Benedetti, H. (1997). The TolB protein interacts with the porins of Escherichia coli. J Bacteriol 179, 7274-7279.

Roberts, D., Hoopes, B. C., McClure, W. R. \& Kleckner, N. (1985). IS10 transposition is regulated by DNA adenine methylation. Cell 43, 117-130.

Shägger, H. \& von Jagow, G. (1987). Tricine-sodium docecyl sulphate-polyacrylamide gel electrophoresis for the separation of proteins in the range of 1 to $100 \mathrm{kDa}$. Anal Biochem 166, 368-379.

Sternberg, N. (1985). Evidence that adenine methylation influences DNA-protein interactions in Escherichia coli. J Bacteriol 164, 490-493.

Torreblanca, J. \& Casadesus, J. (1996). DNA adenine methylase mutants of Salmonella typhimurium and a novel dam-regulated locus. Genetics 144, 15-26.

Torreblanca, J., Marques, S. \& Casadesus, J. (1999). Synthesis of FinP RNA by plasmids F and pSLT is regulated by DNA adenine methylation. Genetics 152, 31-45.

van der Woude, M. W. \& Low, D. A. (1994). Leucine-responsive regulatory protein and deoxyadenosine methylase control the phase variation and expression of the $s f a$ and $d a a$ pili operons in Escherichia coli. Mol Microbiol 11, 605-618.

van der Woude, M., Braaten, B. \& Low, D. (1996). Epigenetic phase variation of the pap operon in Escherichia coli. Trends Microbiol 4, 5-9.

Wai, S. N., Takade, A. \& Amako, K. (1995). The release of outer membrane vesicles from the strains of enterotoxigenic Escherichia coli. Microbiol Immunol 39, 451-456.

Whitmire, W. M. \& Garon, C. F. (1993). Specific and nonspecific 
responses of murine B cells to membrane blebs of Borrelia burgdorferi. Infect Immun 61, 1460-1467.

Yokoyama, K., Horii, T., Yamashino, T., Hashikawa, S., Barua, S., Hasegawa, T., Watanabe, H. \& Ohta, M. (2000). Production of shiga toxin by Escherichia coli measured with reference to the membrane vesicle-associated toxins. FEMS Microbiol Lett $\mathbf{1 9 2}$ $139-144$.
Zhou, L., Srisatjaluk, R., Justus, D. E. \& Doyle, R. J. (1998). On the origin of membrane vesicles in gram-negative bacteria. FEMS Microbiol Lett 163, 223-228.

Received 31 October 2001; revised 12 December 2001; accepted 19 December 2001. 